\title{
Impaired T-cell proliferation among HAART- treated adults with suboptimal CD4 recovery in an African cohort
}

Damalie Nakanjako ${ }^{1,2^{*}}$, Isaac Ssewanyana ${ }^{3}$, Rose Nabatanzi ${ }^{3}$, Agnes Kiragga ${ }^{2}$, Moses R Kamya ${ }^{1}$, Huyen Cao ${ }^{4}$ and Harriet Mayanja-Kizza ${ }^{1}$

\begin{abstract}
Background: Most HIV-infected subjects exhibit a progressive rise in CD4 T-cell counts after initiation of highly active antiretroviral therapy (HAART). However, a subset of individuals exhibit very poor CD4 T-cell recovery despite effective control of HIV-RNA viraemia. We evaluated CD4 T-cell proliferation among suboptimal responders and its correlation with CD4 T-cell activation.

Methods: The magnitude of CD4 increase (difference between absolute CD4 counts at baseline and absolute CD4 counts at 4 years of ART) was grouped into 4 quartiles for the 211 patients with sustained HIV-RNA viral suppression. Cases of 'Suboptimal immune responders' included patients within the lowest quartile [Median CD4 increase 165 (Range -43-298) cells/ $\mu \mathrm{l} ; \mathrm{n}=52$ ] and a comparison group of 'Optimal immune responders' was defined as patients within the highest quartile of CD4 increase [Median CD4 increase 528 (Range 417-878) cells/ $\mu$; $n=52]$. Frozen PBMC were thawed and analysed from a convenient sample of 39 suboptimal responders and 48 optimal responders after 4 years of suppressive antiretroviral therapy. T-cell activation was measured by proportions of T-cells expressing surface marker CD38 and HLADR (CD4+CD38+HLA-DR+ and CD8+CD38+HLA-DR+ cells). T-cell proliferation was determined by the extent of carboxyfluorescein diacetate succinimidyl ester (CFSE) dye dilution on culture day 5 of PBMCs in the presence of antigen (SEB, PPD, CMVpp65, GagA and GagD). Samples were analyzed on a FACS Calibur flow cytometer and flow data was analyzed using FlowJo and GraphPad.
\end{abstract}

Results: Overall, CD4 T-cell proliferation on stimulation with SEB, PPD, CMVpp65, Gag A and Gag D.antigens, was lower among suboptimal than optimal responders; this was significant for SEB (CD4+ $p=0.003$; CD8+ $p=0.048$ ) and PPD antigens (CD8+ $\mathrm{p}=0.038$ ). Among suboptimal responders, T-cell proliferation decreased with increasing immune activation (Negative correlation; slope $=-0.13 \pm-0.11$ ) but not among optimal responders.

Conclusion: T-cell immune activation and exhaustion were associated with poor proliferation among suboptimal responders to HAART despite sustained viral suppression. We recommend studies to further understand the mechanisms leading to impaired T-cell function among suboptimal responders as well as the potential role of immune modulation in optimizing CD4 count and functional recovery after HAART.

Keywords: T-cell proliferation, Immune activation, Suboptimal immune recovery, HAART immune responses, HIV/AIDS

\footnotetext{
* Correspondence: drdamalie@yahoo.com

'Makerere University College of Health Sciences, P.O. Box 7072, Kampala,

Uganda

${ }^{2}$ Infectious Diseases Institute, Makerere University College of Health Sciences,

Kampala, Uganda

Full list of author information is available at the end of the article
}

\section{Biomed Central}

(c) 2013 Nakanjako et al.; licensee BioMed Central Ltd. This is an Open Access article distributed under the terms of the Creative Commons Attribution License (http://creativecommons.org/licenses/by/2.0), which permits unrestricted use, distribution, and reproduction in any medium, provided the original work is properly cited. 


\section{Background}

CD4 T-helper function remains critical to effective immune responses to common infections among HIVinfected individuals. With chronic HIV infection, CD4 Tcell function decreases with HIV-RNA viraemia [1,2]. Evidence suggests that the HIV-associated immune dysfunction is reversible with control of HIV-viraemia [2,3]. Strong HIV-specific immune responses have been observed among individuals receiving potent highly active antiretroviral therapy (HAART) for acute HIV infection [1]. Most HIV-infected subjects exhibit a progressive rise in CD4 Tcell counts after initiation of HAART [4]. However, up to $40 \%$ of HAART-treated individuals exhibit very poor CD4 T-cell recovery despite effective control of HIV-RNA viraemia $[5,6]$. CD4 count measurement is the main laboratory tool for monitoring immune recovery in many HIV treatment programs in sub-Saharan Africa (SSA) [7]. With the increasing number of individuals on HAART for longer period of time and with the emerging population of suboptimal responders to HAART despite viral suppression, there is need to consider CD4 T-cell function recovery as the ultimate measure of immune recovery. In the developed world, significant proliferative responses were observed in $30-69 \%$ of individuals on suppressive HAART [8-11]. There is paucity of data on CD4 T-cell function recovery within HIV treatment programs in SSA. Moreover, persistently low T-cell function is likely to contribute to the increased risk of opportunistic infection observed among the individuals with suboptimal CD4 reconstitution despite suppressive HAART [12]. This study compared T-cell proliferation among suboptimal and optimal responders after four years of suppressive HAART upon in-vitro stimulation with common antigens like Staphylococcus Enterotoxin B, Cytomegalovirus and the M. Tuberculosis antigen purified protein derive (PPD). We also determined the correlation between $\mathrm{T}$-cell proliferation and immune activation among suboptimal responders to HAART. Our results give insight on the persistent immune dysfunction among patients that do not reconstitute their CD4 counts despite long periods of HIV-RNA viral suppression and emphasize the need for innovative immune modulation interventions to optimise immune recovery in this sub-population of HAART-treated individuals.

\section{Methods}

\section{Study setting and participants}

Between April, 2004 and April, 2005, 559 consecutive ARTnaive HIV-infected patients, were initiated on HAART and enrolled into the Infectious Diseases Institute (IDI) prospective observational research cohort as previously described [13]. Patients were initiated on first-line HAART at CD4 counts $\leq 200$ cells/ $\mu$ l according to Ugandan guidelines for HAART initiation at the time. Drugs were provided through the Global Fund (a generic combined formulation of stavudine [d4T], lamivudine [3TC], and nevirapine [NVP] and the US President's Emergency Plan for AIDS Relief ( a combined formulation of zidovudine [ZDV] and 3TC plus efavirenz [EFZ] or NVP). Patients with toxicity to ZDV were changed to tenofovir [TDF]. All patients received cotrimoxazole (or dapsone) prophylaxis according to the national policy to provide cotrimoxazole to all people living with HIV (PLHIV). Adherence to HAART was encouraged by at least 3 individual and group counseling sessions. Patients were reviewed monthly by the study physicians that evaluated among others, adherence to medication, toxicities and acute infections. HIV RNA viral loads, complete blood counts and CD4 lymphocyte counts were measured 6 monthly.

\section{Definition of suboptimal CD4 reconstitution}

Various definitions have been used to describe suboptimal CD4 reconstitution following HAART including the magnitude of the CD4 cell increase [14]. Kaufmann et al. defined suboptimal CD4 reconstitution an absolute CD4 count $<500$ cells/ $\mu \mathrm{l}$ after 5 years of sustained viral loads $<1000$ copies [5]. The authors thought the latter criteria would over-estimate suboptimal immune response in our cohort since less than one-third of all our patients had attained an absolute CD4 count $\geq 500$ cells/ $\mu$ l. Therefore we used a cohort-specific definition of suboptimal CD4 reconstitution in order to consider the whole spectrum of CD4 recovery. The magnitude of CD4 increase (difference between absolute CD4 counts at baseline and absolute CD4 counts after 4 years of HAART), for the 211 patients with sustained HIV-RNA viral suppression, was grouped into 4 quartiles. Cases of 'Suboptimal immune responders' included patients within the lowest quartile [Median CD4 increase 165 (Range -43-298) cells/ $\mu \mathrm{l} ; \mathrm{n}=52$ ] and the comparison group of 'Optimal immune responders' included patients within the highest quartile of CD4 increase [Median CD4 increase 528 (Range 417-878) cells/ $\mu \mathrm{l} ; \mathrm{n}=52$ ]. This study compared CD4 T-cell proliferation among suboptimal and optimal responders. The second and third quartiles, including 104 individuals with a median CD4 increase 282 (Range 200415) cells $/ \mu$ l were considered as average immune responders and were not included in this study which looked at the two extremes of immune recovery. Frozen PBMCs were from a convenient sample of 39 suboptimal responders and 48 optimal responders after 4 years of suppressive HAART (see Figure 1).

We excluded patients that had an opportunistic infection in the previous 6 months. Rapid tests for malaria antigens, stool microscopy for intestinal helminths and a C-reactive protein were done to exclude common causes of immune activation in our setting. Written informed consent was obtained from all participants. This study was approved by national review research and ethics board; the Uganda National Council for Science and Technology. 


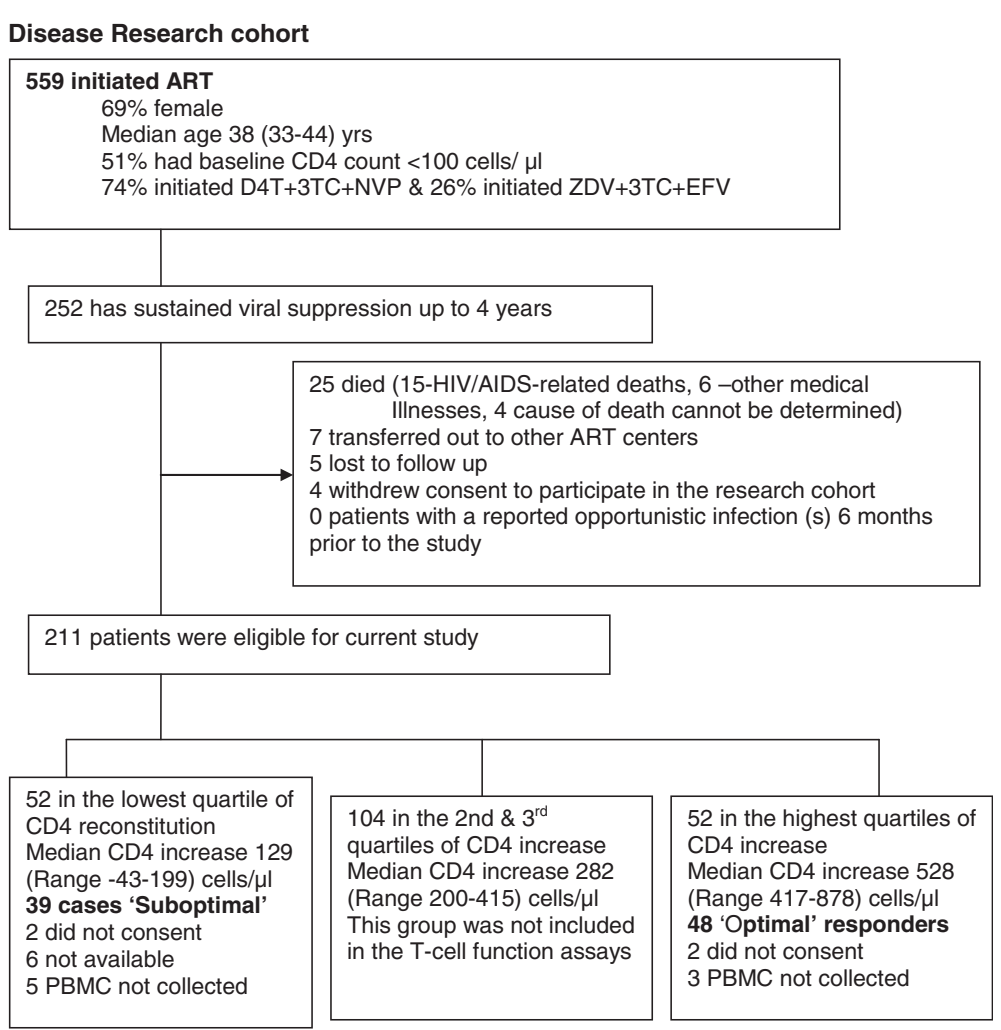

Figure 1 Profile of patients on antiretroviral therapy within the Infectious Diseases Institute Research cohort.

The immunology assays were performed at the Cytotoxic T-lymphocyte laboratory (CTL) at the Joint Clinical Research Center (JCRC), Kampala, Uganda.

\section{Peripheral Blood Mononuclear Cells (PBMC) separation}

Fifty (50) mls of whole blood, collected in ACD-A bottles, was processed for PBMC separation within 4 hours of collection. PBMCs were separated by Ficoll-Hypaque density configuration, washed and re-suspended in phosphate buffer saline (PBS) containing heat inactivated fetal calf serum (FCS). PBMCs were frozen and stored in Fetal Calf Serum with $10 \%$ dimethyl sulfoxide (DMSO), in liquid nitrogen until assay time. On day 0 , cell surface staining was done with the following antibodies; CD3 APC, CD4 PerCP-Cy5 .5, HLA-DR FITC and CD38 PE for immune activation and PD-1 APC for immune exhaustion (BD Biosciences San Jose, CA). Samples were analyzed on a FACS Calibur flow cytometer (BD Biosciences, San Jose, CA). Overall, at least 50,000 events in the CD3-positive gate were collected. The gating was standardised and set using fluorescence minus one controls for HLADR and CD38.

\section{T-cell proliferation assays}

Cell proliferation was determined by carboxyfluorescein diacetate succinimidyl ester (CFSE) dilution using the CellTrace $^{\text {tu }}$ CFSE Cell Proliferation Kit (Invitrogen,
Carlsbad, CA), per the manufacturer's instructions. Previously thawed PBMC, which had been rested overnight were cultured in the presence of either, Staphylococcal enterotoxin B (SEB) Sigma-Aldrich, St,Lois, MO, Purified Protein Derivative (PPD), Q Biogene Carlsbad, CA, USA, CMVpp65- $\mu \mathrm{L}$ (Q Biogene Carlsbad, CA, USA), Gag A and Gag D (NIH/NIAID repository) or un stimulated at $37^{\circ} \mathrm{C}$ and $5 \% \mathrm{CO}_{2}$, for five days. The final concentration of the antigens was $1 \mu \mathrm{g} / \mathrm{ml}$ of PBMC (1 million PBMC per well), as previously optimised by our team [15]. On day 5, the cells were harvested and stained with CD3 APC, CD4 PE, CD8 PerCp-Cy5.5 (BD Biosciences, San Jose, CA). The flowcytometry data analysis was performed using FlowJo software (TreeStar, Version X). Only data with a minimum of 10,000 acquired events of $\mathrm{CD} 3+\mathrm{CD} 4+$ or $\mathrm{CD} 3+\mathrm{CD} 8+$ were analyzed. T-cell proliferation was determined by the extent of carboxyfluorescein diacetate succinimidyl ester (CFSE) dye dilution on culture day 5 of thawed PBMCs in the presence of antigen (PPD, CMVpp65, GagA and GagD). Staphylococcal Enterotoxin B (SEB Sigma-Aldrich, St. Louis, MO) stimulation was used as a positive control. All the study participants demonstrated significant proliferation following SEB stimulation. Proliferation results $\leq$ background response (un stimulated PBMC) were considered negative whereas results $\geq$ twice the background values (after subtraction of background) 
were considered positive. Figure 2 shows the gating strategy we used to analyse T-cell proliferation using CFSE dye and SEB antigen stimulation.

\section{Statistical analysis}

Using Prism Graph Pad 5.0 software, we compared proportions of proliferated CD4+ and CD8+ T-cells among optimal and suboptimal responders; using the Mann Whitney test for non-parametric variables. $P$ values $<0.05$ were considered statistically significant. We also determined the linear correlation between T-cell proliferation and immune activation (co-expression of CD38 and HLADR cell surface markers).

\section{Results}

\section{Study participants}

After 4 years of follow up on HAART, 252/559 (45\%) patients had sustained HIV-RNA viral suppression. Of these, 41 were excluded due to the following reasons; death $(n=25)$, lost to follow-up $(n=5)$, voluntary request

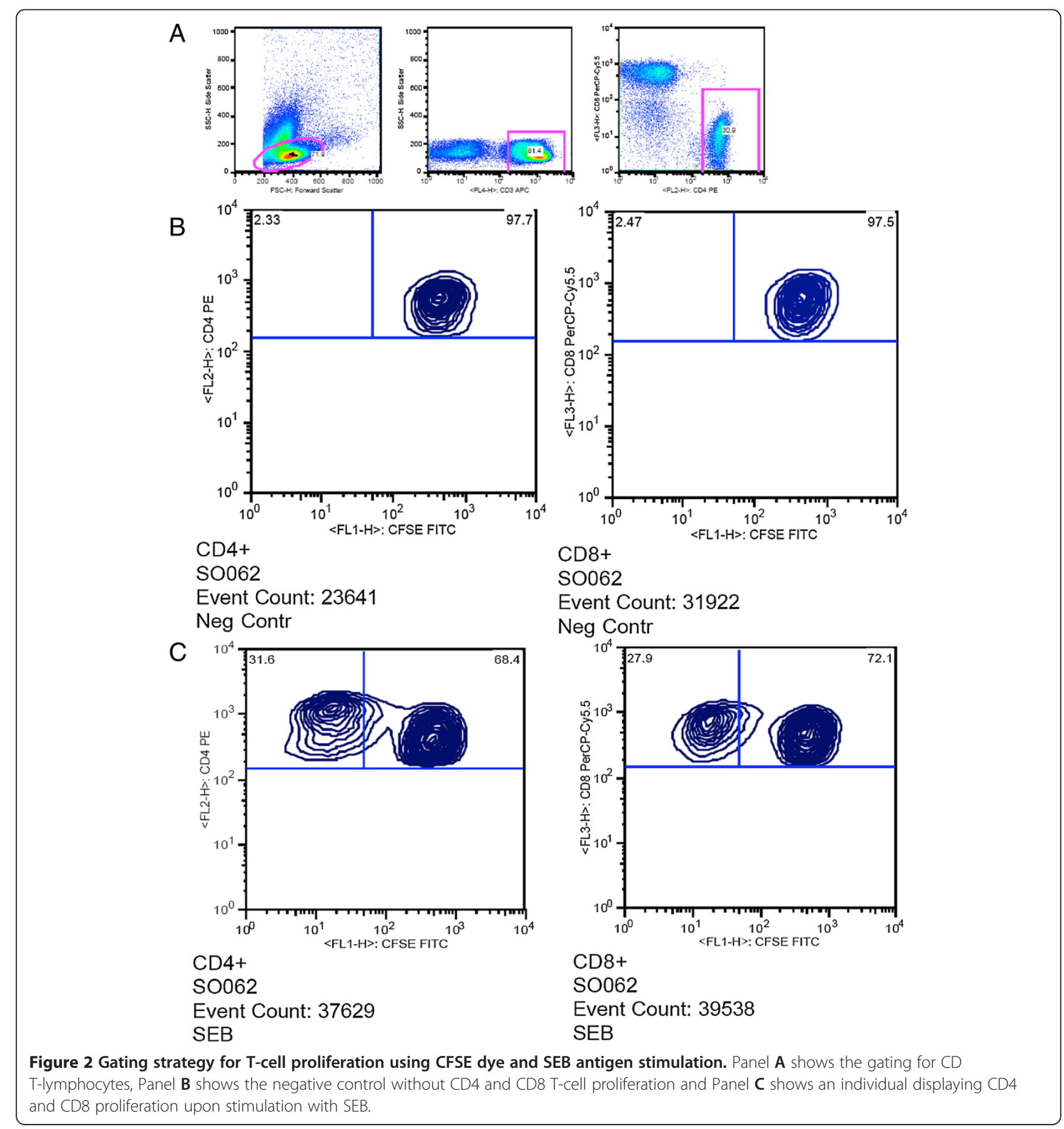


to transfer to and voluntary termination from the study $(\mathrm{n}=11)$. The median age [Inter Quartile Range (IQR)], was 36 (31-42) years, Body Mass Index (BMI); 22 (IQR 20-72), and haemoglobin level, 13 (IQR 12-14) g/dl.

\section{Socio-demographic characteristics of optimal and suboptimal responders}

Age, gender and HAART regimen were similar among optimal and suboptimal responders ( $\mathrm{p}$ value $=0.290$, 0.062 and 0.340 respectively. Suboptimal responders had lower CD4 counts at the time of HAART initiation as wells as years after therapy (see Table 1).

\section{T-cell proliferation after day 5 of stimulation with SEB,} PPD, GagA\&D and CMVpp65 antigens

Figure 3 shows our typical analysis of T-cell proliferation of PBMC stained labelled with fluorescent dye 5,6carboxyfluorescein diacetate succinimidyl ester (CFSE); on day 5 of stimulation with SEB, Gag A \& D, PPD and CMV antigens. Upon stimulation with SEB, a super-antigen, Tcell proliferation was lower among suboptimal responders than optimal responders to suppressive HAART; (CD4+ $\mathrm{P}=0.003$ and $\mathrm{CD} 8+\mathrm{P}=0.048$ ). Similarly, $\mathrm{T}$-cell proliferation upon stimulation with PPD was lower among suboptimal and optimal responders and the difference was stronger for CD8 T-cells (CD4+; $\mathrm{p}=0.136$ and CD8+; $\mathrm{p}=0.038$ ); see Figure 4. We found a negative correlation between immune activation and CD4 T-cell proliferation among HAART-treated adults (slope -0.13); as well as a negative correlation between CD8 T-cell exhaustion and proliferation, among suboptimal responders (see Figure 5).

\section{Discussion}

Within the context of sustained viral suppression after 4 years of HAART, we found that suboptimal responders exhibit lower levels of T-cell proliferation than optimal responders. We showed significant differences when PBMCs were stimulated with SEB and PPD. Our results are consistent with previous reports of impaired in-vitro HIV-specific CD4 T-cell proliferation among HIV-infected cells although these were not in a setting of suppressive HAART[2,16,17]. In addition to HIV-specific responses, this study analysed T-cell responses to common antigen (SEB, PPD, CMVpp65) to which majority of HIV-infected patients are exposed. Our findings imply that despite sustained viral suppression, the persistently low CD4 Tcell function is likely to predispose suboptimal responders to opportunistic infections and severe Staphylococcus aureus infections. This could explain previous reports that HAART-treated individuals with poor CD4 recovery despite viral suppression were at increased risk of opportunistic infections and mortality [12]. Given the low proliferation levels among suboptimal responders, we postulate that suboptimal responders could potentially benefit from longer periods of cotrimoxazole prophylaxis for opportunistic infections as well as additional vaccines of common bacterial infections such as pneumococcal vaccines. With the increasing numbers of individuals on HAART in sub-Saharan Africa, there is clearly need for prospective studies to understand therapeutic interventions for the emerging population of $30-40 \%$ suboptimal responders despite viral suppression $[12,18]$ in order to maximise immune recovery during first-line antiretroviral therapy.

Table 1 Characteristics of 128 patients with sustained viral suppression after 4 years of antiretroviral therapy at the Infectious Diseases Institute research cohort

\begin{tabular}{|c|c|c|c|}
\hline $\begin{array}{l}\text { Median CD4 increase } \varphi \\
\text { median (range) cells/ } \mu \mathrm{l}\end{array}$ & $\begin{array}{l}\text { Suboptimal CD4 responders } \\
\quad(n=39) 165(-43-298) ;]\end{array}$ & $\begin{array}{l}\text { Optimal CD4 reconstitution } \\
(n=48) 528 \text { (Range 417-878) }\end{array}$ & $P$ value* \\
\hline Age (yrs) [median (IQR)] & $37(31-42)$ & $35(31-43)$ & 0.875 \\
\hline Female gender [n (\%)] & $28(72)$ & $22(46)$ & 0.062 \\
\hline Baseline CD4 count cells/ $\mu \mathrm{l}$ [median (IQR)] $¥$ & $78(2-117)$ & 115 (97-185) & 0.018 \\
\hline 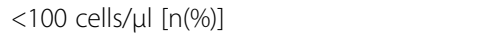 & $26(67)$ & $7(15)$ & 0.0015 \\
\hline Current CD4 cells/ $\mu \mathrm{l}$ [median (IQR)] & $214(190-282)$ & $474(438-645)$ & $<0.001$ \\
\hline BMI [median (IQR)] & $22(20-25)$ & $26(22-28)$ & 0.162 \\
\hline Hemoglobin [median (IQR)] & $14(12-15)$ & $14(13-15)$ & 0.481 \\
\hline Hepatitis B positive [n] & 2 & 1 & 0.911 \\
\hline \multicolumn{4}{|l|}{ First-line HAART regimen $\infty$} \\
\hline D4T-3TC-NVP/EFZ [n (\%)] & $12(31)$ & $27(56)$ & \\
\hline ZDV-3TC-NVP/EFZ [n (\%)] & $25(64)$ & $17(35)$ & \\
\hline TDF-3TC-NVP/EFZ [n (\%)] & $2(5)$ & $4(8)$ & 0.340 \\
\hline
\end{tabular}

*Chi square test was used for categorical variables and Kruskal-Wallis test was used for the continuous variables.

$\varphi$ The magnitude of CD4 increase from the baseline counts was grouped into 4 quartiles; 'Suboptimal responders' were patients within the lowest quartile and 'Optimal responders' were individuals within the highest quartile of CD4 increase.

$¥$ All patients initiated antiretroviral therapy at CD4 200 cells/ $\mu \mathrm{l}$ and below.

$\infty$ Study participants were all on their initial first-line regimen given that they had sustained viral suppression 

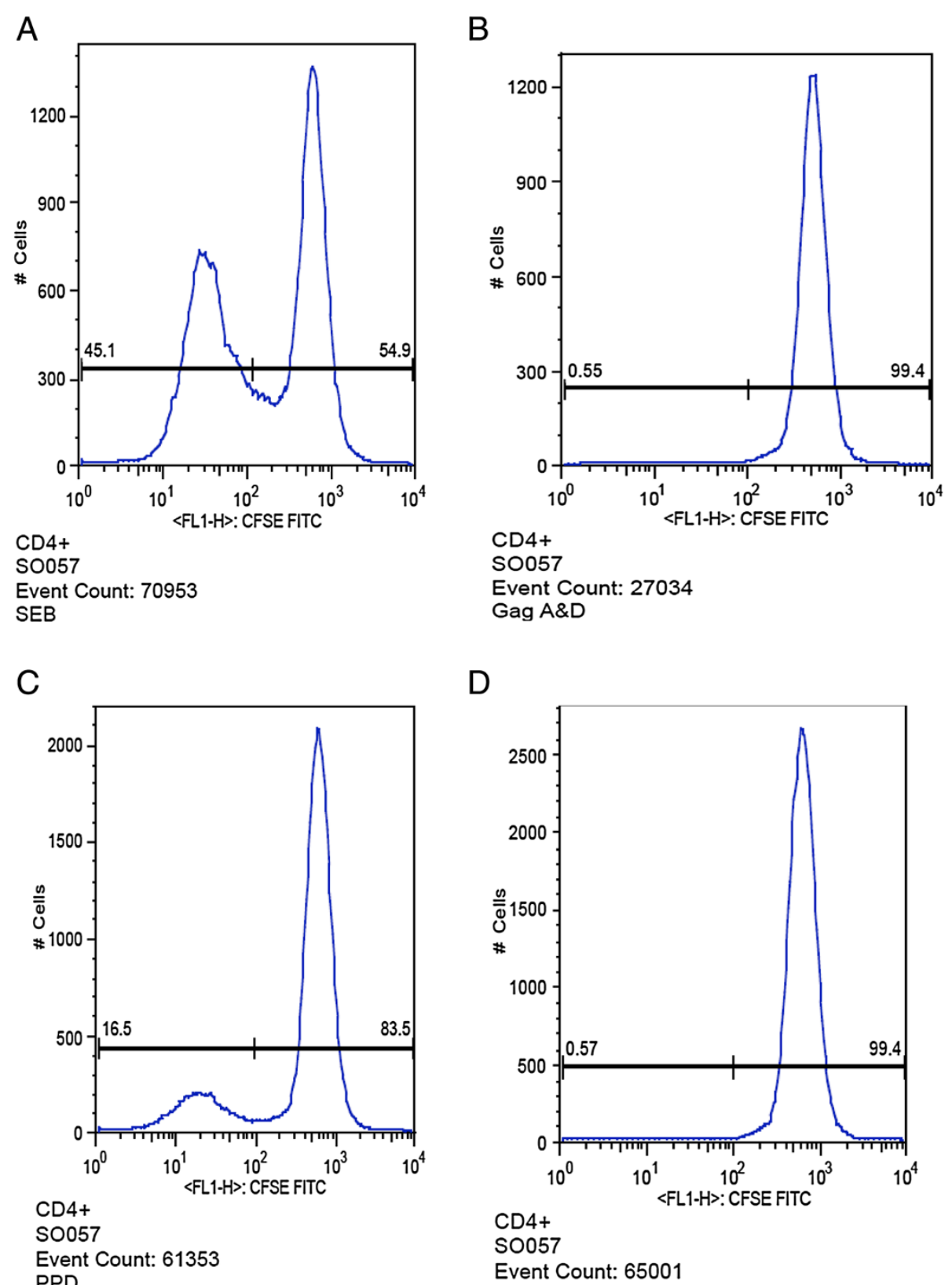

D

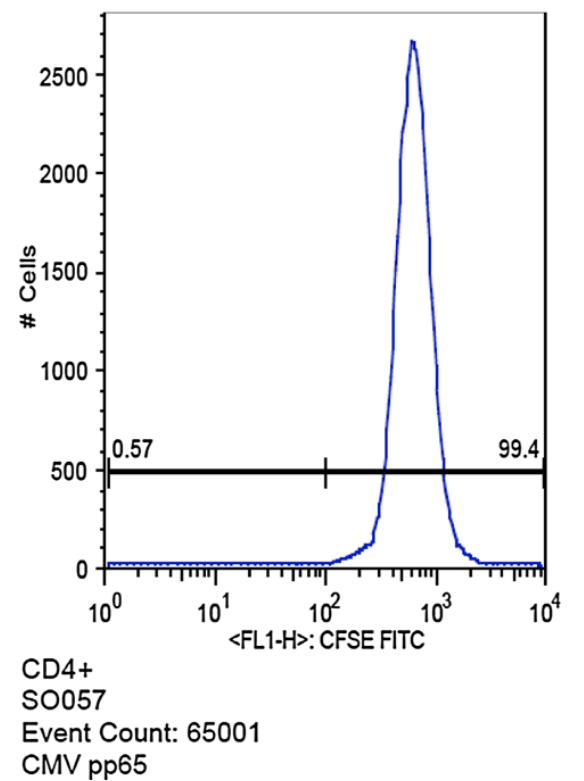

Figure 3 Typical analysis of T-cell proliferation of peripheral blood mononuclear cells (PBMC) stained labelled with fluorescent dye 5,6carboxyfluorescein diacetate succinimidyl ester (CFSE); on day 5 of stimulation with SEB, Gag A \& D, PPD and CMV antigens.

Panels A-D show the typical presentation of T-cell proliferation up stimulation with SEB, Gag A \& D, PPD and CMV antigens respectively.

The mechanisms of functional impairment of surviving CD4 and CD8 T-cells during chronic HIV infection need to be understood to support the development of targeted interventions. For example, Boasso et al. show that Indoleanine 2,3 dioxyenase (IDO), an immunosuppressive enzyme, plays a role in inhibition of T-cell proliferation and its in-vitro inhibition increased CD4 T-cell responses in PBMCs from HIV-infected patients [16]. Immune activation-induced fibrosis of lymphoid tissue in advanced HIV disease is thought to contribute to depletion of naive T-cells and increased apoptosis and subsequently perpetuate cumulative loss of T-cells that may persist despite antiretroviral therapy $[19,20]$. In addition, recent evidence from mouse studies suggests that lymphoid tissue inducer cells are a relatively newly recognised family of innate lymphoid cells that may have a role in CD4 T-cell responses [21]. Furthermore, HIVinfected dendritic cells produce gp120 that contributes to impaired CD4 T-cell immune responses in-vivo; therefore 


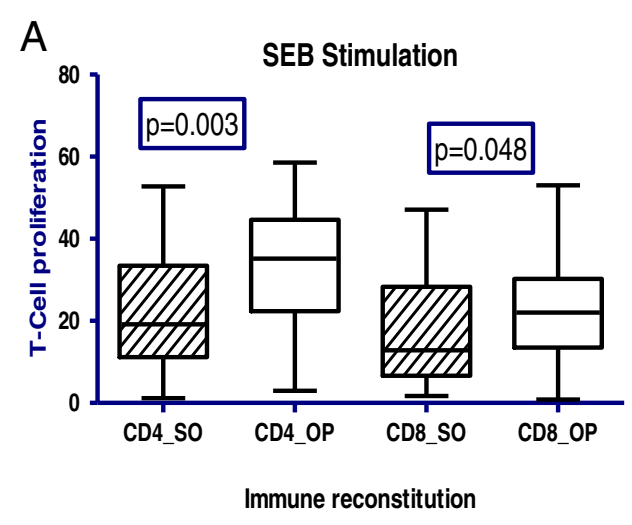

C

Gag A\&D

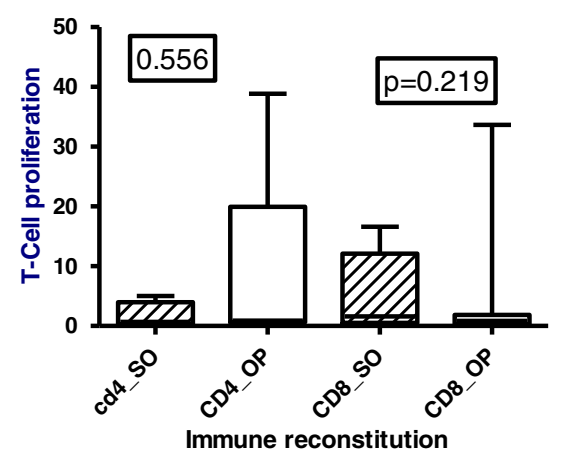

B

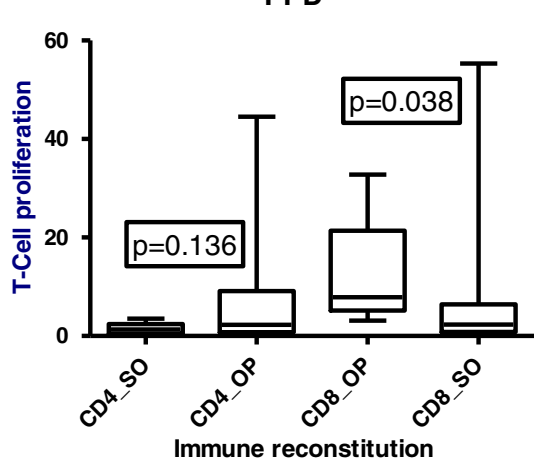

D

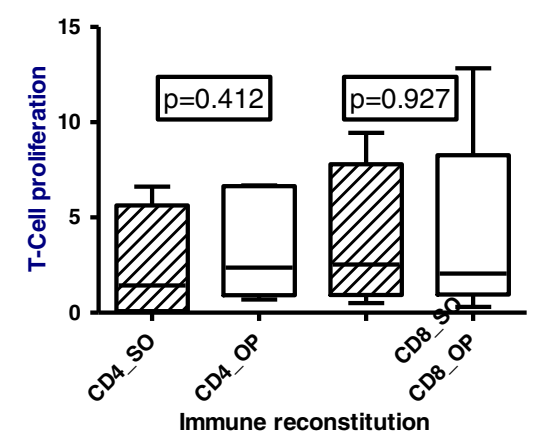

Figure 4 CD4 and CD8 T-cell proliferation up stimulation by SEB, PPD, GagA\&D and CMV antigens respectively; among suboptimal (SO) relative to optimal (OP) immune responders after $\mathbf{4}$ years of suppressive antiretroviral therapy. Panel A shows lower CD4 and CD8 proliferation among suboptimal relative to optimal responders upon SEB stimulation, Panel B shows lower CD8 proliferation among suboptimal relative to optimal responders upon stimulation with tuberculin PPD, Panel C shows lower CD4 proliferation upon stimulation with Gag A \& D although the difference is not statistically significant and Panel $\mathbf{D}$ shows lower CD4 proliferation upon stimulation with CMV although the difference is not statistically significant.
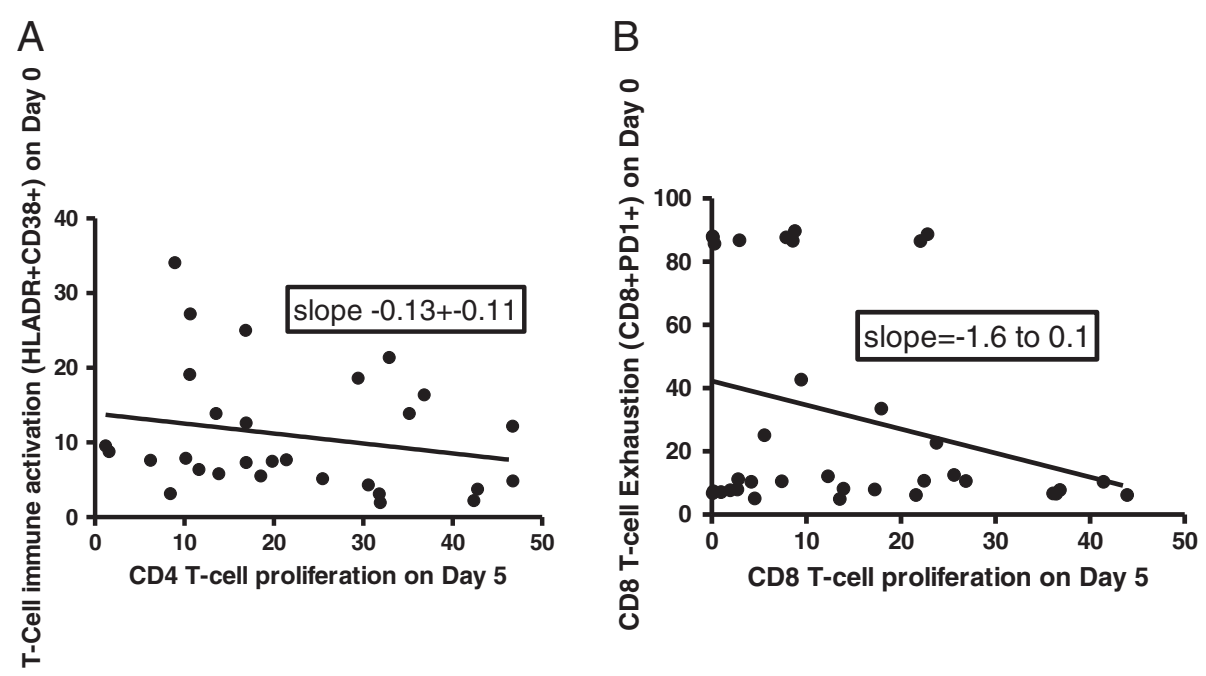

Figure 5 Correlation of immune activation and exhaustion with T-cell proliferation among suboptimal responders to HAART in a Ugandan cohort. Panel A shows that CD4 T-cell proliferation decreases with increasing levels of immune activation and Panel B shows that CD8 T-cell proliferation reduces with increasing levels of exhaustion. 
agents that block the gp120-mediated immune suppression could potentially modulate immune responses in HIVinfected individuals [17] and their role in HAART-treated adults may be worth investigation. There is clearly a need to understand and explore the role of these mechanisms in CD4 T-cell function recovery within the setting of longterm suppressive HAART. This study contributes to the increasing evidence that monitoring of immune activation levels, viral loads and $\mathrm{CD} 4$ function recovery are relevant to compliment the current widely used CD4 count monitoring $[3,22]$ in order to optimise long-term immune recovery within HIV treatment programs in SSA.

We found that CD4 T-cell proliferation decreased with increasing levels of immune activation. Although the exact mechanism is yet to be understood, this data is consistent with our previous report that immune activation and exhaustion were significantly associated with suboptimal CD4 recovery [18]. Similarly, CD8 T-cell proliferation reduced with increasing immune exhaustion. We hypothesize that in addition to low T-cell proliferative ability, the persistently high immune activation and exhaustion levels drive the inflammatory death pathways and subsequently the impairment in CD4 count and functional recovery [23]; however the reverse could also be true. We therefore need to further understand the prevalent T-cell death pathways among suboptimal responders with persistent immune activation.

Our findings are consistent with previous follow-up studies in European and American cohorts that reported failure in long-term restoration of numeric CD4 counts, with the recovery process reaching a plateau after 4-5 years of therapy [5,24-26]. The patients in this study had been on treatment for 4 years which is the likely period for the plateau phenomenon. There is need for followup data on specific T-cell responses from this HAART cohort to provide the trends of CD4 count and functional recovery beyond 5 years of therapy. It is also important to note that the patients we studied had initiated HAART in the advanced stages of HIV disease which might be a contributing factor to suboptimal immune recovery $[5,25,26]$ due to the collagen deposition and fibrosis of lymphoid tissue during later stages of HIV infection $[4,19,27]$. It will be interesting to perform similar assays among patients that initiate HAART at higher CD4 counts ( $>350$ cells as per 2012 national antiretroviral therapy guidelines) or during acute HIV infection, which associated with enhanced likelihood of CD4 count recovery [28]. Due to the limitations of the four color flowcytometry used in this study, we were not able to describe all the T-cell subsets including the memory Tcells of the specific antigens used. This would probably provide more insight into the mechanism of suboptimal immune recovery. For example, previous studies, among HAART-treated individuals with CMV retinitis, showed that memory CMV-specific CD4 and CD8 T-cells expanded several years after HAART initiation [29].

To date, there is no consensus on when and how to treat immunological failure in a setting of viral suppression. Suboptimal responders pose several clinical questions, including questions about the clinical risk associated with persistent immunodeficiency and about possible interventions to optimise clinical and immunological benefits of HAART $[12,30]$. From a clinical point of view, it is reasonable to hypothesise that adjuvant therapy with anti-immune activation agents [31,32] may improve T-cell proliferation potential among suboptimal responders. Our work presents an interface between clinical care and basic science research. We also add to the evidence that immune modulation could potentially maximize immunological recovery during suppressive antiretroviral therapy.

\section{Conclusion}

CD4 T-cell proliferation was lower among suboptimal than optimal responders, and reduced with increasing levels of CD4 T-cell immune activation markers. T-cell immune activation and exhaustion were associated with poor proliferation among suboptimal responders to HAART despite sustained viral suppression. We recommend prospective studies to understand long-term CD4 numeric and functional recovery among suboptimal responders. In addition, we recommend innovative clinical studies to understand the effect of immune modulants on T-cell function recovery among the emerging populations of suboptimal responders.

\section{Competing interests \\ The authors declare that they have no competing interests.}

\section{Authors' contributions}

DN made substantial contribution to the conception, design, data collection, analysis and drafting of the manuscript. IS contributed to the immune assays, data analysis and interpretation. HMK contributed to the conception, design, data interpretation and revision of the manuscript. AK made substantial contribution to the study design and the statistical analysis. RN contributed to the data collection and MRK contributed to the conception, design, data interpretation and revision of the manuscript. All authors read and approved the final manuscript. $\mathrm{HC}$ made substantial contribution to the conception, design, immune assays, data analysis, interpretation and revision of the manuscript. All authors read and approved the final manuscript

\section{Acknowledgement}

The authors acknowledge the staff of the Adult Infectious Disease Clinic research cohort for supporting the data collection, the CTL laboratory at the Joint Clinic Research Center where the assays were performed. In addition, the authors acknowledge the study participants for accepting to participate in this study. The work was supported by the Gilead-funded Sewankambo clinical research scholarship program at the Infectious Diseases Institute (IDI), Makerere University College of Health Sciences and the Wellcome Trust-

funded Makerere University Infection and Immunity post-doctoral program.

\section{Author details}

'Makerere University College of Health Sciences, P.O. Box 7072, Kampala, Uganda. ${ }^{2}$ Infectious Diseases Institute, Makerere University College of Health Sciences, Kampala, Uganda. ${ }^{3}$ Joint Clinical Research Center, Kampala, Uganda. ${ }^{4}$ California Department of Public Health, Richmond, CA, USA. 
Received: 19 December 2012 Accepted: 18 June 2013

Published: 20 June 2013

\section{References}

1. Rosenberg ES, Billingsley JM, Caliendo AM, Boswell SL, Sax PE, Kalams SA Walker BD: Vigorous HIV-1-specific CD4+ T cell responses associated with control of viremia. Science 1997, 278(5342):1447-1450.

2. Iyasere C, Tilton JC, Johnson AJ, Younes S, Yassine-Diab B, Sekaly RP, Kwok WW, Migueles SA, Laborico AC, Shupert WL, et al: Diminished proliferation of human immunodeficiency virus-specific CD4+ T cells is associated with diminished interleukin-2 (IL-2) production and is recovered by exogenous IL-2. J Viro/ 2003, 77(20):10900-10909.

3. Glencross DK, Janossy G, Coetzee LM, Lawrie D, Scott LE, Sanne I, McIntyre JA, Stevens W: CD8/CD38 activation yields important clinical information of effective antiretroviral therapy: findings from the first year of the CIPRA-SA cohort. Cytometry B Clin Cytom 2008, 74(Suppl 1):S131-140.

4. Guihot A, Bourgarit A, Carcelain G, Autran B: Immune reconstitution after a decade of combined antiretroviral therapies for human immunodeficiency virus. Trends Immunol 2011, 32(3):131-137.

5. Kaufmann GR, Furrer $H$, Ledergerber B, Perrin L, Opravil M, Vernazza $P$, Cavassini M, Bernasconi E, Rickenbach M, Hirschel B, et al: Characteristics, determinants, and clinical relevance of CD4 T cell recovery to $<500$ cells/ microL in HIV type 1-infected individuals receiving potent antiretroviral therapy. Clin Infect Dis 2005, 41(3):361-372.

6. Nakanjako D, Kiragga A, Ibrahim F, Castelnuovo B, Kamya MR, Easterbrook PJ: Sub-optimal CD4 reconstitution despite viral suppression in an urban cohort on antiretroviral therapy (ART) in sub-Saharan Africa: frequency and clinical significance. AIDS Res Ther 2008, 5:23.

7. Nakanjako D, Colebunders R, Coutinho AG, Kamya MR: Strategies to optimize HIV treatment outcomes in resource-limited settings. AIDS Rev 2009, 11(4):179-189.

8. Lange CG, Valdez H, Medvik K, Asaad R, Lederman MM: CD4+ Tlymphocyte nadir and the effect of highly active antiretroviral therapy on phenotypic and functional immune restoration in HIV-1 infection. Clin Immunol 2002, 102(2):154-161.

9. Blankson JN, Gallant JE, Siliciano RF: Proliferative responses to human immunodeficiency virus type 1 (HIV-1) antigens in HIV-1-infected patients with immune reconstitution. J Infect Dis 2001, 183(4):657-661.

10. Markowitz M, Jin X, Hurley A, Simon V, Ramratnam B, Louie M, Deschenes GR, Ramanathan M Jr, Barsoum S, Vanderhoeven J, et al: Discontinuation of antiretroviral therapy commenced early during the course of human immunodeficiency virus type 1 infection, with or without adjunctive vaccination. J Infect Dis 2002, 186(5):634-643.

11. Palmer BE, Boritz E, Blyveis N, Wilson CC: Discordance between frequency of human immunodeficiency virus type 1 (HIV-1)-specific gamma interferon-producing CD4(+) T cells and HIV-1-specific lymphoproliferation in HIV-1-infected subjects with active viral replication. J Virol 2002, 76(12):5925-5936.

12. Gazzola L, Tincati C, Bellistri GM, Monforte A, Marchetti G: The absence of CD4+ T cell count recovery despite receipt of virologically suppressive highly active antiretroviral therapy: clinical risk, immunological gaps, and therapeutic options. Clin Infect Dis 2009, 48(3):328-337.

13. Kamya MR, Mayanja-Kizza H, Kambuqu A, Bakeera-Kitaka S, Semitala F, MwebazeSonga P, Castelnuovo B, Schaefer P, Spacek LA, Gasasira AF, et al: Predictors of long-term viral failure among ugandan children and adults treated with antiretroviral therapy. J Acquir Immune Defic Syndr 2007, 46(2):187-193.

14. Teixeira L, Valdez H, McCune JM, Koup RA, Badley AD, Hellerstein MK, Napolitano LA, Douek DC, Mbisa G, Deeks S, et al: Poor CD4 T cell restoration after suppression of HIV-1 replication may reflect lower thymic function. AIDS 2001, 15(14):1749-1756

15. Kityo C, Bousheri S, Akao J, Ssali F, Byaruhanga R, Ssewanyana I, Muloma P Myalo S, Magala R, Lu Y, et al: Therapeutic immunization in HIV infected Ugandans receiving stable antiretroviral treatment: a Phase I safety study. Vaccine 2011, 29(8):1617-1623.

16. Boasso A, Herbeuval JP, Hardy AW, Anderson SA, Dolan MJ, Fuchs D, Shearer GM: HIV inhibits CD4+ T-cell proliferation by inducing indoleamine 2,3-dioxygenase in plasmacytoid dendritic cells. Blood 2007, 109(8):3351-3359.

17. Kawamura T, Gatanaga H, Borris DL, Connors M, Mitsuya H, Blauvelt A Decreased stimulation of CD4+ T cell proliferation and IL-2 production by highly enriched populations of HIV-infected dendritic cells. J Immunol 2003, 170(8):4260-4266.
18. Nakanjako D, Ssewanyana I, Mayanja-Kizza H, Kiragga A, Colebunders R, Manabe YC, Nabatanzi R, Kamya MR, Cao H: High T-cell immune activation and immune exhaustion among individuals with suboptimal CD4 recovery after 4 years of antiretroviral therapy in an African cohort. BMC Infect Dis 2011, 11:43.

19. Zeng M, Smith AJ, Wietgrefe SW, Southern PJ, Schacker TW, Reilly CS, Estes $J D$, Burton GF, Silvestri G, Lifson JD, et al: Cumulative mechanisms of lymphoid tissue fibrosis and T cell depletion in HIV-1 and SIV infections. J Clin Invest 2011, 121(3):998-1008.

20. Zeng M, Southern PJ, Reilly CS, Beilman GJ, Chipman JG, Schacker TW, Haase AT: Lymphoid tissue damage in HIV-1 infection depletes naive T cells and limits T cell reconstitution after antiretroviral therapy. PLOS Pathog 2012, 8(1):e1002437.

21. Lane PJ, Gaspal FM, McConnell FM, Kim MY, Anderson G, Withers DR: Lymphoid tissue inducer cells: innate cells critical for CD4+ T cell memory responses? Ann N Y Acad Sci 2012, 1247:1-15.

22. Moodley K, Coetzee LM, Glencross DK: Decentralised CD38 activation monitoring: aspects of practical implementation and standardisation. $J$ Immunol Methods 2012, 378(1-2):121-127.

23. Negredo E, Massanella M, Puig J, Perez-Alvarez N, Gallego-Escuredo JM, Villarroya J, Villarroya F, Molto J, Santos JR, Clotet B, et al: Nadir CD4 T cell count as predictor and high CD4 T cell intrinsic apoptosis as final mechanism of poor CD4 T cell recovery in virologically suppressed HIV-infected patients: clinical implications. Clin Infect Dis 2010, 50(9):1300-1308.

24. Mocroft A, Phillips AN, Ledergerber B, Katlama C, Chiesi A, Goebel FD, Knysz $B$, Antunes F, Reiss P, Lundgren JD: Relationship between antiretrovirals used as part of a CART regimen and CD4 cell count increases in patients with suppressed viremia. AIDS 2006, 20(8):1141-1150.

25. Moore RD, Keruly JC: CD4+ cell count 6 years after commencement of highly active antiretroviral therapy in persons with sustained virologic suppression. Clin Infect Dis 2007, 44(3):441-446.

26. Le Moing V, Thiebaut R, Chene G, Sobel A, Massip P, Collin F, Meyohas M, Al Kaied F, Leport C, Raffi F: Long-term evolution of CD4 count in patients with a plasma HIV RNA persistently $<500$ copies $/ \mathrm{mL}$ during treatment with antiretroviral drugs. HIV Med 2007, 8(3):156-163.

27. Brenchley JM, Schacker TW, Ruff LE, Price DA, Taylor JH, Beilman GJ, Nguyen PL, Khoruts A, Larson M, Haase AT, et al: CD4+ T cell depletion during all stages of HIV disease occurs predominantly in the gastrointestinal tract. J Exp Med 2004, 200(6):749-759.

28. Le T, Wright EJ, Smith DM, He W, Catano G, Okulicz JF, Young JA, Clark RA, Richman DD, Little SJ, et al: Enhanced CD4+ T-cell recovery with earlier HIV-1 antiretroviral therapy. N Engl J Med 2013, 368(3):218-230.

29. Sacre K, Carcelain G, Cassoux N, Fillet AM, Costagliola D, Vittecoq D, Salmon D, Amoura Z, Katlama C, Autran B: Repertoire, diversity, and differentiation of specific CD8 T cells are associated with immune protection against human cytomegalovirus disease. J Exp Med 2005, 201(12):1999-2010.

30. WHO: Guidelines for the use of antiretroviral agents in HIV-1 infected adults and adolescents: Management of the treatment experienced patient; Virologic and Immunological failure; 2010. http://www.who.int/hiv/pub/arv/adult2010/ en/index.htm.

31. Lori F, De Forni D, Katabira E, Baev D, Maserati R, Calarota SA, Cahn P, Testori M, Rakhmanova A, Stevens MR: VS411 reduced immune activation and HIV-1 RNA levels in 28 days: randomized proof-of-concept study for AntiViral-HyperActivation limiting therapeutics. PLoS One 2012, 7(10):e47485.

32. Piconi S, Parisotto S, Rizzardini G, Passerini S, Terzi R, Argenteri B, Meraviglia P, Capetti A, Biasin M, Trabattoni D, et al: Hydroxychloroquine drastically reduces immune activation in HIV-infected, antiretroviral therapy-treated immunologic nonresponders. Blood 2011, 118(12):3263-3272.

doi:10.1186/1471-2172-14-26

Cite this article as: Nakanjako et al:: Impaired T-cell proliferation among

HAART-treated adults with suboptimal CD4 recovery in an African cohort. BMC Immunology 2013 14:26. 